\title{
Characterization of absolute and uniform continuity
}

\author{
Ismail Aslan*1 (D), Oktay Duman² (D) \\ ${ }^{1}$ Hacettepe University, Department of Mathematics, Çankaya TR-06800, Ankara, Turkey \\ ${ }^{2}$ TOBB University of Economics and Technology, Department of Mathematics, Sögütözü TR-06530, \\ Ankara, Turkey
}

\begin{abstract}
In the present paper, by considering nonlinear integral operators and using their approximations via regular summability methods, we obtain characterizations for some function spaces including the space of absolutely continuous functions, the space of uniformly continuous functions, and their other variants. We observe that Bell-type summability methods are quite effective to generalize and improve some related results in the literature. At the end of the paper, we discuss some special cases and applications.
\end{abstract}

Mathematics Subject Classification (2010). 26A45, 26A46, 26B30, 41A35, 47G10

Keywords. summability process, absolute continuity, uniform continuity, nonlinear integral operators, bounded variation

\section{Introduction}

Integral operators of convolution type and Mellin type are widely used in approximation theory. Some of them have a linear structure (see [14,19-21,28]) while others are nonlinear (see [2-4]). Beside approximation theory, there are many engineering applications of these operators, such as in optical physics, image processing and signal processing (see $[13,17,22,23])$. They also play a crucial role in the characterization of absolutely continuous functions (see [5-7]). In our recent papers, we also consider regular summability methods instead of the usual convergence in the approximation by these operators (see [9-11]) in order to generalize and improve the known results in the literature. We should note that, in the approximation by these operators, we consider suitable functions spaces endowed with the variation semi-norm, the uniform (supremum) norm, the $L_{p}$ norm, and so on.

In mathematical analysis, a summability method is an alternative formulation of convergence of a series or a sequence which is divergent in the conventional sense. So, it is quite useful to overcome the divergence problem in some kind of divergent series or sequences. It is also possible to accelerate the rate of convergence by using a suitable summability method (see [30,33]). Furthermore, so far many applications of summability methods have been studied in approximation theory (see [1,8,12, 29,31]).

Since, in this work, we mainly consider Bell-type summability (see $[15,16]$ ), we first recall this concept as follows.

\footnotetext{
*Corresponding Author.

Email addresses: ismail-aslan@hacettepe.edu.tr (I. Aslan), oduman@etu.edu.tr (O. Duman)

Received: 02.07.2019; Accepted: 13.11.2019
} 
Let $\mathcal{A}=\left\{A^{v}\right\}:=\left\{\left[a_{n k}^{v}\right]\right\}(n, k, v \in \mathbb{N})$ be a family of infinite matrices of real or complex numbers. Then, for a sequence $x=\left\{x_{k}\right\}_{k \in \mathbb{N}}, \mathcal{A}$-transform of $x$ is denoted by $\mathcal{A} x:=\left\{(\mathcal{A} x)_{n}^{v}\right\}$, which is given by $(\mathcal{A} x)_{n}^{v}=\sum_{k=1}^{\infty} a_{n k}^{v} x_{k}(n, v \in \mathbb{N})$ if the series is convergent for every $n, v$. Then, we say that $x$ is $\mathcal{A}$-summable to a number $L$ if $\lim _{n \rightarrow \infty}(\mathcal{A} x)_{n}^{v}=L$ uniformly in $v \in \mathbb{N}$, which is denoted by

$$
\mathcal{A}-\lim x=L .
$$

In particular, we focus on nonnegative regular summability methods satisfying the property $\mathcal{A}-\lim x=L$ whenever $\lim x=L$. Note that a method $\mathcal{A}=\left\{A^{v}\right\}$ is called row finite if, for every $v \in \mathbb{N}$, each row of the matrix $A^{v}$ contains at most a finite number of nonzero terms.

Observe that Bell-type summability methods are quite general and contain many wellknown methods, such as, the Cesàro mean, the almost convergence and the order summability (see $[18,24-27]$ ).

In this work, our goal is, using summability methods, to give some characterizations for absolute continuity and uniform continuity, which will be more general than the results by Angeloni and Vinti [2,6]. For this process, we use nonlinear integral operators of convolution type and Mellin type, which are defined in the next sections. We also discuss some significant applications and special cases of our results.

\section{Characterizations of absolute continuity}

In this section, we obtain characterizations of the absolute continuity. By using the periodicity of functions, we first get a characterization in one dimension. Later, without the periodicity, we give characterizations in $N$-dimension with the help of the approximation by nonlinear integral operators constructed with respect to the Lebesgue measure and the Haar measure.

Throughout the section, let $\mathcal{A}=\left\{\left[a_{n k}^{v}\right]\right\}(n, k, v \in \mathbb{N})$ be a nonnegative regular summability method.

We now study the above cases in the following three subsections.

\subsection{Characterization in one dimension with periodicity}

We first need the following function spaces:

- $B V_{2 \pi}$, the space of all $2 \pi$-periodic measurable functions of bounded variation on the interval $[-\pi, \pi]$.

- $A C_{2 \pi}$, the space of all $2 \pi$-periodic and absolutely continuous functions on the interval $[-\pi, \pi]$.

- $L_{2 \pi}^{1}$, the space of all $2 \pi$-periodic and Lebesgue integrable functions.

Then, we consider the nonlinear integral operators introduced in [10] (see also $[2,3]$ in the case of $\mathcal{A}=\{I\}$, the identity matrix):

$$
\mathcal{T}_{n, v}(f ; s)=\sum_{k=1}^{\infty} a_{n k}^{v} T_{k}(f ; s),
$$

where

$$
T_{k}(f ; s)=\int_{-\pi}^{\pi} K_{k}(t, f(s-t)) d t .
$$

We assume that $f$ is a $2 \pi$-periodic measurable function for which the series in (2.1) is well-defined. The kernel $K_{k}: \mathbb{R} \times \mathbb{R} \rightarrow \mathbb{R}$ is a family of measurable functions such that $K_{k}(t, s)=L_{k}(t) H_{k}(s)$ for every $s, t \in \mathbb{R}$, where $L_{k} \in L_{2 \pi}^{1}$ and the functions $H_{k}: \mathbb{R} \rightarrow \mathbb{R}$ with $H_{k}(0)=0$ have the uniform Lipschitz property, i.e., there exists a constant $C>0$ such that $\left|H_{k}(x)-H_{k}(y)\right| \leq C|x-y|$ for every $x, y \in \mathbb{R}$ and $k \in \mathbb{N}$.

Then, assuming the following conditions 
(i) There exists a constant $M>0$ such that $\sup _{n, v \in \mathbb{N}} \sum_{k=1}^{\infty} a_{n k}^{v}\left\|L_{k}\right\|_{2 \pi}=M<\infty$,

(ii) $\mathcal{A}-\lim \left(\int_{-\pi}^{\pi} L_{k}(t) d t\right)=1$,

(iii) for any fixed $\delta>0, \mathcal{A}-\lim \left(\int_{|t| \geq \delta}\left|L_{k}(t)\right| d t\right)=0$,

(iv) $\lim _{k \rightarrow \infty} \frac{V_{J}\left[G_{k}\right]}{m(J)}=0$ uniformly with respect to every bounded interval $J \subset \mathbb{R}$, where $G_{k}(u):=H_{k}(u)-u, m(J)$ is the length of the interval $J$ and $V_{J}$ denotes the total variation on $J$,

we proved in [10] that, for every $f \in A C_{2 \pi}$,

$$
\lim _{n \rightarrow \infty} V_{2 \pi}\left[\mathcal{T}_{n, v}(f)-f\right]=0 \text { uniformly in } v \in \mathbb{N}
$$

holds, which generalizes the result in $[2,3]$.

Remark 2.1. Our operators $\mathcal{T}_{n, v}$ in (2.1) are defined by using regular matrix transformations of the classical operators $T_{k}$ in $(2.2)$ introduced by Angeloni and Vinti (see $[2,3]$ ). Observe that conditions $(i),(i i)$ and (iii) for the operators $T_{k}$ are more general than the ones in $[2,3]$. However, in this subsection, we prove that it is still possible to characterize the absolute continuity under these conditions for the operators $T_{k}$. Furthermore, the limit given for the sequence $\left\{\mathcal{T}_{n, v}\right\}$ does not need to exist for the sequence $\left\{T_{k}\right\}$ (see Example 4.1 . Of course, in order to cover the classical conditions in $[2,3]$ we especially use the regular summability methods since our conditions are then satisfied at once due to the regularity of the methods.

We also need the following condition on the kernel $L_{k} \in L_{2 \pi}^{1}$.

For every $n, v \in \mathbb{N}$ and for all $\varepsilon>0$, there exists a $\delta>0$ such that for every nonoverlapping subintervals $\left\{\left[\alpha_{i}, \beta_{i}\right]\right\}_{i=1}^{m}$ of $[-\pi, \pi]$,

$$
\sum_{i=1}^{m}\left(\beta_{i}-\alpha_{i}\right)<\delta \text { implies } \sum_{i=1}^{m} \sum_{k=1}^{\infty} a_{n k}^{v}\left|L_{k}\left(\beta_{i}\right)-L_{k}\left(\alpha_{i}\right)\right|<\varepsilon .
$$

Remark 2.2. We should note that, in particular, if $\mathcal{A}$ is a row finite method and also if $L_{k} \in A C_{2 \pi}$, then we immediately get (2.4). Such kernels and row finite methods will be given in Section 4. Furthermore, if we take $\mathcal{A}=\{I\}$, then (2.4) is equivalent to the absolute continuity of $L_{k}$. Hence, our condition (2.4) generalizes the absolute continuity of $L_{k}$ by a nonnegative regular summability method.

Now we get the next lemma.

Lemma 2.3. Assume that (2.4) holds. If $f \in B V_{2 \pi}$, then $\mathcal{T}_{n, v}(f) \in A C_{2 \pi}$ for every $n, v \in \mathbb{N}$.

Proof. Since $L_{k}$ is $2 \pi$-periodic, by the substitution $s-t=z$ we may write from (2.1) that

$$
\mathcal{T}_{n, v}(f ; z)=\sum_{k=1}^{\infty} a_{n k}^{v} \int_{-\pi}^{\pi} L_{k}(s-z) H_{k}(f(z)) d z
$$

By assumption, for every $\varepsilon>0$ there exists a $\delta>0$ such that, for every nonoverlapping subintervals $\left\{\left[\alpha_{i}, \beta_{i}\right]\right\}_{i=1}^{m}$ of $[-\pi, \pi],(2.4)$ holds whenever $\sum_{i=1}^{m}\left(\beta_{i}-\alpha_{i}\right)<\delta$. Using $H_{k}(0)=0$ and the Fubini-Tonelli theorem, we get the following inequality

$$
\begin{aligned}
\sum_{i=1}^{m}\left|\mathcal{T}_{n, v}\left(f ; \beta_{i}\right)-\mathcal{T}_{n, v}\left(f ; \alpha_{i}\right)\right| & \leq C \sum_{i=1}^{m} \sum_{k=1}^{\infty} a_{n k}^{v} \int_{-\pi}^{\pi}\left|L_{k}\left(\beta_{i}-z\right)-L_{k}\left(\alpha_{i}-z\right)\right||f(z)| d z \\
& =C \int_{-\pi}^{\pi} \sum_{i=1}^{m} \sum_{k=1}^{\infty} a_{n k}^{v}\left|L_{k}\left(\beta_{i}-z\right)-L_{k}\left(\alpha_{i}-z\right)\right||f(z)| d z
\end{aligned}
$$


where $C$ is the Lipschitz constant of $H_{k}$. Since $\sum_{i=1}^{m}\left(\beta_{i}-z-\left(\alpha_{i}-z\right)\right)<\delta$ and $(2.4)$ is satisfied, we conclude that

$$
\sum_{i=1}^{m}\left|\mathcal{T}_{n, v}\left(f ; \beta_{i}\right)-\mathcal{T}_{n, v}\left(f ; \alpha_{i}\right)\right| \leq C\|f\|_{1} \varepsilon,
$$

where the symbol $\|\cdot\|_{1}$ denotes the usual norm on $L_{2 \pi}^{1}$. The last inequality immediately gives that $\mathcal{T}_{n, v}(f) \in A C_{2 \pi}$.

Then, our first characterization theorem is as follows.

Theorem 2.4. Let $f \in B V_{2 \pi}$ and assume that conditions $(i)-(i v)$ and (2.4) hold. Then,

$$
f \in A C_{2 \pi} \Leftrightarrow \lim _{n \rightarrow \infty} V_{2 \pi}\left[\mathcal{T}_{n, v}(f)-f\right]=0 \text { uniformly in } v \text {. }
$$

Proof. The necessity immediately follows from Theorem 2.3 of [10]. For the sufficiency, assume that

$$
\lim _{n \rightarrow \infty} V_{2 \pi}\left[\mathcal{T}_{n, v}(f)-f\right]=0
$$

holds. Then, by Lemma 2.3, we get $\mathcal{T}_{n, v} f \in A C_{2 \pi}$. On the other hand, since $A C_{2 \pi}$ is a closed subspace of $B V_{2 \pi}$ with respect to variation semi-norm (see Lemma 2.1 of [14]), we observe that $f$ must belong to $A C_{2 \pi}$.

\subsection{Characterization in $N$-dimension (without periodicity) with respect to the Lebesgue measure}

In this part, we adopt Tonelli's definition for the $N$-dimensional bounded variation (see [32]). Here we use the following notations and definitions.

- $L^{1}\left(\mathbb{R}^{N}\right)$, the space of all Lebesgue integrable functions on $\mathbb{R}^{N}$ with the usual norm $\|\cdot\|_{1}$.

- $|\mathbf{x}|$, the Euclidean norm of the $N$-dimension vector $\mathbf{x}=\left(x_{1}, \ldots, x_{N}\right) \in \mathbb{R}^{N}$.

- For a given vector $\mathbf{x}=\left(x_{1}, x_{2}, \ldots, x_{N}\right) \in \mathbb{R}^{N}$, the $(N-1)$ dimensional vector $x_{j}^{\prime}$ is obtained from $\mathbf{x}$ by removing the $j$-th coordinate of $\mathbf{x}$, which is given by

$$
x_{j}^{\prime}:=\left(x_{1}, x_{2}, \ldots, x_{j-1}, x_{j+1}, \ldots, x_{N}\right) \in \mathbb{R}^{N-1} .
$$

Then, we write $\mathbf{x}=\left(x_{j}^{\prime}, x_{j}\right)$.

- For an interval $I=\prod_{i=1}^{N}\left[a_{i}, b_{i}\right]$, we denote $(N-1)$-dimensional interval by $I_{j}^{\prime}=$ $\left[a_{j}^{\prime}, b_{j}^{\prime}\right]$ which is obtained by deleting the $j$-th coordinate from $I$, i.e., $I=\left[a_{j}^{\prime}, b_{j}^{\prime}\right] \times$ $\left[a_{j}, b_{j}\right], j=1, \ldots, N$.

- For a given function $f: \mathbb{R}^{N} \rightarrow \mathbb{R}$, we define

$$
\Phi_{j}(f, I):=\int_{a_{j}^{\prime}}^{b_{j}^{\prime}} V_{\left[a_{j}, b_{j}\right]}\left[f\left(x_{j}^{\prime}, \cdot\right)\right] d x_{j}^{\prime} \quad \text { for } j=1, \ldots, N,
$$

where $V_{\left[a_{j}, b_{j}\right]}\left[f\left(x_{j}^{\prime}, \cdot\right)\right]$ is the usual one dimensional (Jordan) variation of the $j$-th section of $f$, namely the function $g_{j}\left(x_{j}\right):=f\left(x_{j}^{\prime}, x_{j}\right)$.

- Let

$$
\Phi(f, I):=\left\{\sum_{j=1}^{N} \Phi_{j}^{2}(f, I)\right\}^{\frac{1}{2}} .
$$

Then $\Phi(f, I)=\infty$ if $\Phi_{j}(f, I)=\infty$ for some $j=1, \ldots, N$. 
- The definition of the variation of $f$ on an interval $I \subset \mathbb{R}^{N}$ is given by

$$
V_{I}[f]:=\sup \sum_{q=1}^{m} \Phi\left(f, J_{q}\right),
$$

where the supremum is taken over all the families of $N$-dimensional subintervals $\left\{J_{1}, \ldots, J_{m}\right\}$ which are partitions of $I$.

- Let $V[f]:=\sup _{I \subset \mathbb{R}^{N}} V_{I}[f]$, where the supremum being taken over all the intervals $I \subset \mathbb{R}^{N}$. If $V[f]<\infty$, we say that a function $f \in L^{1}\left(\mathbb{R}^{N}\right)$ is of bounded variation over $\mathbb{R}^{N}$

- $B V\left(\mathbb{R}^{N}\right)$, the space of all functions of bounded variation over $\mathbb{R}^{N}$. In this space, $\|f\|_{B V}:=V[f]$ denotes the variation semi-norm of $f$.

- $A C_{\text {loc }}\left(\mathbb{R}^{N}\right)$, the space of all functions $f: \mathbb{R}^{N} \rightarrow \mathbb{R}$ with locally absolutely continuous in Tonelli's sense, i.e., for any $N$-dimensional interval $I=\prod_{j=1}^{N}\left[a_{j}, b_{j}\right] \subset \mathbb{R}^{N}$ if, for every $j=1, \ldots, N$, and for every $\varepsilon>0$, there exists a $\delta>0$ such that for almost every $s_{j}^{\prime} \in \mathbb{R}^{N-1}$ and for all finite collections of non-overlapping intervals $\left[\alpha_{j}^{\rho}, \beta_{j}^{\rho}\right] \subset\left[a_{j}, b_{j}\right], \rho=1, \ldots, \lambda$,

$$
\sum_{\rho=1}^{\lambda}\left(\beta_{j}^{\rho}-\alpha_{j}^{\rho}\right)<\delta \text { implies } \sum_{\rho=1}^{\lambda}\left|f\left(s_{j}^{\prime}, \beta_{j}^{\rho}\right)-f\left(s_{j}^{\prime}, \alpha_{j}^{\rho}\right)\right|<\varepsilon .
$$

- $A C\left(\mathbb{R}^{N}\right):=B V\left(\mathbb{R}^{N}\right) \cap A C_{\text {loc }}\left(\mathbb{R}^{N}\right)$.

Now consider the following nonlinear integral operators defined in [11] (see also [5, 6] in the case of $\mathcal{A}=\{I\})$ :

$$
\mathcal{T}_{n, v}(f ; \mathbf{s})=\sum_{k=1}^{\infty} a_{n k}^{v} T_{k}(f ; \mathbf{s}),
$$

where

$$
T_{k}(f ; \mathbf{s})=\int_{\mathbb{R}^{N}} K_{k}(\mathbf{t}, f(\mathbf{s}-\mathbf{t})) d \mathbf{t}
$$

We assume that $f: \mathbb{R}^{N} \rightarrow \mathbb{R}$ is measurable and bounded on $\mathbb{R}^{N}$ for which the series in (2.5) is well-defined. We also suppose that $K_{k}(\mathbf{t}, s): \mathbb{R}^{N} \times \mathbb{R} \rightarrow \mathbb{R}$ satisfies $K_{k}(\mathbf{t}, s)=$ $L_{k}(\mathbf{t}) H_{k}(s)$ for every $\mathbf{t} \in \mathbb{R}^{N}$ and $s \in \mathbb{R}$, where $\left\{L_{k}\right\} \subset L^{1}\left(\mathbb{R}^{N}\right)$ and $H_{k}$ is uniformly Lipschitz with $H_{k}(0)=0$.

We know from [11] that, for a given $f \in A C\left(\mathbb{R}^{N}\right)$, in order to get the approximation

$$
\lim _{n \rightarrow \infty} V\left[\mathcal{T}_{n, v}(f)-f\right]=0 \text { uniformly in } v
$$

we need the following conditions:

(i) $)^{\prime}$ There exists a constant $M>0$ such that $\sup _{n, v \in \mathbb{N}} \sum_{k=1}^{\infty} a_{n k}^{v}\left\|L_{k}\right\|_{1}=M<\infty$,

$(\text { ii) })^{\prime} \mathcal{A}-\lim \left(\int_{\mathbb{R}^{N}} L_{k}(\mathbf{t}) d \mathbf{t}\right)=1$,

(iii)' for any fixed $\delta>0, \mathcal{A}-\lim \left(\int_{|\mathbf{t}| \geq \delta} L_{k}(\mathbf{t}) d \mathbf{t}\right)=0$,

and condition (iv) stated in Subsection 2.1.

We should remark that the limit in (2.7) does not need to exist if we replace the sequence $\left\{\mathcal{T}_{n, v}(f)\right\}$ in (2.5) with the sequence $\left\{T_{k}(f)\right\}$ in (2.6) (see Example 4.2).

To give a characterization of absolute continuity we also need the following assumption on the kernel $L_{k}$. 
For every $n, v \in \mathbb{N}$ and $\varepsilon>0$, there exists a $\delta>0$ such that for any $N$-dimensional interval $I=\prod_{i=1}^{N}\left[a_{i}, b_{i}\right]$ and for every collection of nonoverlapping intervals $\left\{\left[\alpha_{j}^{\rho}, \beta_{j}^{\rho}\right]\right\}_{\rho=1}^{\lambda}$ of the interval $\left[a_{j}, b_{j}\right]$

$$
\sum_{\rho=1}^{\lambda}\left(\beta_{j}^{\rho}-\alpha_{j}^{\rho}\right)<\delta \text { implies } \sum_{\rho=1}^{\lambda} \sum_{k=1}^{\infty} a_{n k}^{v}\left|L_{k}\left(s_{j}^{\prime}, \beta_{j}^{\rho}\right)-L_{k}\left(s_{j}^{\prime}, \alpha_{j}^{\rho}\right)\right|<\varepsilon
$$

for every $j=1, \ldots, N$.

Lemma 2.5. Assume that (2.8) holds. If $f \in B V\left(\mathbb{R}^{N}\right)$, then $\mathcal{T}_{n, v}(f) \in A C\left(\mathbb{R}^{N}\right)$ for every $n, v \in \mathbb{N}$.

Proof. It follows from the substitution $\mathbf{s}-\mathbf{t}=\mathbf{z}$ that

$$
\mathcal{T}_{n, v}(f ; \mathbf{z})=\sum_{k=1}^{\infty} a_{n k}^{v} \int_{\mathbb{R}^{N}} L_{k}(\mathbf{s}-\mathbf{z}) H_{k}(f(\mathbf{z})) d \mathbf{z} .
$$

Using (2.8) and the Fubini-Tonelli theorem we get

$$
\begin{aligned}
& \sum_{\rho=1}^{\lambda}\left|\mathcal{T}_{n, v}\left(f ;\left(s_{j}^{\prime}, \beta_{j}^{\rho}\right)\right)-\mathcal{T}_{n, v}\left(f ;\left(s_{j}^{\prime}, \alpha_{j}^{\rho}\right)\right)\right| \\
& \leq C \sum_{\rho=1}^{\lambda} \sum_{k=1}^{\infty} a_{n k}^{v} \int_{\mathbb{R}^{N}}\left|L_{k}\left(\mathbf{s}_{j}^{\prime}-z_{j}^{\prime}, \beta_{j}^{\rho}-z_{j}\right)-L_{k}\left(\mathbf{s}_{j}^{\prime}-z_{j}^{\prime}, \alpha_{j}^{\rho}-z_{j}\right)\right||f(\mathbf{z})| d \mathbf{z} \\
& \leq C \int_{\mathbb{R}^{N}} \sum_{\rho=1}^{\lambda} \sum_{k=1}^{\infty} a_{n k}^{v}\left|L_{k}\left(\mathbf{s}_{j}^{\prime}-z_{j}^{\prime}, \beta_{j}^{\rho}-z_{j}\right)-L_{k}\left(\mathbf{s}_{j}^{\prime}-z_{j}^{\prime}, \alpha_{j}^{\rho}-z_{j}\right)\right||f(\mathbf{z})| d \mathbf{z} \\
& <C\|f\|_{1} \varepsilon
\end{aligned}
$$

holds due to

$$
\sum_{\rho=1}^{\lambda}\left(\beta_{j}^{\rho}-z_{j}-\left(\alpha_{j}^{\rho}-z_{j}\right)\right)<\delta
$$

This shows that $\mathcal{T}_{n, v}(f) \in A C_{l o c}\left(\mathbb{R}^{N}\right)$. On the other hand, from Lemma 2.1 of [11] we know that $\mathcal{T}_{n, v}(f) \in B V\left(\mathbb{R}^{N}\right)$; and hence $\mathcal{T}_{n, v}(f) \in A C\left(\mathbb{R}^{N}\right)$.

Then, we get the next result.

Theorem 2.6. Let $f \in B V\left(\mathbb{R}^{N}\right)$ and assume that conditions $(i)^{\prime}-(i i i)^{\prime}$, (iv) and (2.8) hold. Then,

$$
f \in A C\left(\mathbb{R}^{N}\right) \Leftrightarrow \lim _{n \rightarrow \infty} V\left[\mathcal{T}_{n, v}(f)-f\right]=0 \text { uniformly in } v \text {. }
$$

Proof. The necessity part is clear from Theorem 2.3 of [11]. Now assume that

$$
\lim _{n \rightarrow \infty} V\left[\mathcal{T}_{n, v}(f)-f\right]=0 \text { uniformly in } v
$$

holds. We know from [14] that $A C\left(\mathbb{R}^{N}\right)$ is a closed subspace of $B V\left(\mathbb{R}^{N}\right)$ with respect to variation semi-norm. Combining this fact with Lemma 2.5 , one can easily conclude that $f \in A C\left(\mathbb{R}^{N}\right)$.

\subsection{Characterization in $N$-dimension (without periodicity) with respect to the Haar measure}

In this part, we use the following notations and definitions. But now, we consider the Haar measure instead of the Lebesgue measure in the definition of the integral operators. 
- The Haar measure of a set $X \subset \mathbb{R}_{+}^{N}$ is defined by

$$
\mu(X)=\int_{X} d \mu:=\int_{X} \frac{d \mathbf{t}}{\langle\mathbf{t}\rangle},
$$

where $\mu$ represents the Haar measure and $\langle\mathbf{t}\rangle:=t_{1} t_{2} \ldots t_{N}$. We remark that (Haar) measure of a set $X$ is invariant under multiplication.

- $L_{\mu}^{1}\left(\mathbb{R}_{+}^{N}\right)$, the space of all functions $f: \mathbb{R}_{+}^{N} \rightarrow \mathbb{R}$ such that

$$
\|f\|_{L_{\mu}^{1}}:=\int_{\mathbb{R}_{+}^{N}}|f(\mathbf{t})| \frac{d \mathbf{t}}{\langle\mathbf{t}\rangle}<\infty .
$$

- $\mathbb{R}_{+}^{N}:=\left\{\left(x_{1}, \ldots, x_{N}\right): x_{i}>0\right.$ for $\left.i=1, \ldots, N\right\}, \mathbf{1}=(1, \ldots, 1)$ is the unit vector of $\mathbb{R}_{+}^{N}$ and $\left\langle x_{j}^{\prime}\right\rangle:=\Pi_{i=1, i \neq j}^{N} x_{i}$.

- $V[f]$ denotes the bounded variation of $f \in L_{\mu}^{1}\left(\mathbb{R}_{+}^{N}\right)$.

- $B V_{\mu}\left(\mathbb{R}_{+}^{N}\right)$, the space of all functions $f \in L_{\mu}^{1}\left(\mathbb{R}_{+}^{N}\right)$ such that $V[f]<\infty$.

- $A C_{\text {loc }}\left(\mathbb{R}_{+}^{N}\right)$, the space of all functions $f: \mathbb{R}_{+}^{N} \rightarrow \mathbb{R}$ such that, for any $N$-dimension interval $I=\prod_{i=1}^{N}\left[a_{i}, b_{i}\right] \subset \mathbb{R}_{+}^{N}$ and for every $i=1, \ldots, N$, the section $g_{j}:\left[a_{j}, b_{j}\right] \rightarrow$ $\mathbb{R}$ is absolutely continuous for almost all vectors $x_{j}^{\prime} \in\left[a_{j}^{\prime}, b_{j}^{\prime}\right]$.

- For a given $\varepsilon>0$, assume that

$$
\sum_{\rho=1}^{\lambda}\left|f\left(s_{j}^{\prime}, \beta_{j}^{\rho}\right)-f\left(s_{j}^{\prime}, \alpha_{j}^{\rho}\right)\right|<\varepsilon
$$

holds for almost every $s_{j}^{\prime} \in \mathbb{R}_{+}^{N-1}$ and for all finite collections of non-overlapping interval $\left[\alpha_{j}^{\rho}, \beta_{j}^{\rho}\right] \subset\left[a_{j}, b_{j}\right], \rho=1, \ldots, \lambda$, whenever

$$
\sum_{\rho=1}^{\lambda}\left(\log \left(\beta_{j}^{\rho}\right)-\log \left(\alpha_{j}^{\rho}\right)\right)<\delta .
$$

In this case, we say that $f$ is log-absolutely continuous on $\mathbb{R}_{+}^{N}$. From Proposition 3.5 of [5], we see that the concepts of absolute continuity and log-absolute continuity are equivalent on $\mathbb{R}_{+}^{N}$.

- $A C_{\mu}\left(\mathbb{R}_{+}^{N}\right):=B V_{\mu}\left(\mathbb{R}_{+}^{N}\right) \cap A C_{\text {loc }}\left(\mathbb{R}_{+}^{N}\right)$.

We now consider the following Mellin-type operators:

$$
\mathcal{T}_{n, v}(f ; \mathbf{s})=\sum_{k=1}^{\infty} a_{n k}^{v} T_{k}(f ; \mathbf{s}),
$$

where

$$
T_{k}(f ; \mathbf{s})=\int_{\mathbb{R}_{+}^{N}} K_{k}(\mathbf{t}, f(\mathbf{s t})) \frac{d \mathbf{t}}{<\mathbf{t}\rangle} .
$$

In (2.10), st $:=\left(s_{1} t_{1}, \ldots, s_{N} t_{N}\right)$ and $f$ is essentially bounded with respect to the Haar measure. We should note that these operators were defined in [9], which generalize the ones in [6]. Here $K_{k}(\mathbf{t}, s): \mathbb{R}_{+}^{N} \times \mathbb{R} \rightarrow \mathbb{R}, K_{k}(\mathbf{t}, s)=L_{k}(\mathbf{t}) H_{k}(s)$ for every $\mathbf{t} \in \mathbb{R}_{+}^{N}$ and $s \in \mathbb{R}$ such that $L_{k} \in L_{\mu}^{1}\left(\mathbb{R}_{+}^{N}\right)$ and $H_{k}$ is uniformly Lipschitz with $H_{k}(0)=0$.

In [9] we proved that, for every $f \in A C_{\mu}\left(\mathbb{R}_{+}^{N}\right)$,

$$
\lim _{n \rightarrow \infty} V\left[\mathcal{T}_{n, v}(f)-f\right]=0 \text { uniformly in } v
$$

provided that the following conditions hold:

(i) $)^{\prime \prime}$ There exists a constant $A>0$ such that $\sup _{n, v \in \mathbb{N}} \sum_{k=1}^{\infty} a_{n k}^{v}\left\|L_{k}\right\|_{L_{\mu}^{1}}=A<\infty$, 
(ii) $)^{\prime \prime} \mathcal{A}-\lim \left(\int_{\mathbb{R}_{+}^{N}} L_{k}(\mathbf{t}) \frac{d \mathbf{t}}{\langle\mathbf{t}\rangle}\right)=1$,

$(\text { iii) })^{\prime \prime}$ for any fixed $0<\delta<1, \mathcal{A}-\lim \left(\int_{|\mathbf{1}-\mathbf{t}|>\delta} L_{k}(\mathbf{t}) \frac{d \mathbf{t}}{\langle\mathbf{t}\rangle}\right)=0$,

and the same condition (iv) in Subsection 2.1.

Note that the limit in (2.11) does not need to be valid for the sequence $\left\{T_{k}(f)\right\}$ in (2.10).

For the characterization theorem, we also need the following assumption.

For very $n, v \in \mathbb{N}$ and for every $\varepsilon>0$ there exists a $\delta>0$ such that for any $N$ dimensional interval $I=\prod_{i=1}^{N}\left[a_{i}, b_{i}\right] \subset \mathbb{R}_{+}^{N}$, and for every collection of non-overlapping intervals $\left\{\left[\alpha_{j}^{\rho}, \beta_{j}^{\rho}\right]\right\}_{\rho=1}^{\lambda}$ of the interval $\left[a_{j}, b_{j}\right]$,

$$
\sum_{\rho=1}^{\lambda}\left|\log \left(\beta_{j}^{\rho}\right)-\log \left(\alpha_{j}^{\rho}\right)\right|<\delta \text { implies } \sum_{\rho=1}^{\lambda} \sum_{k=1}^{\infty} a_{n k}^{v}\left|L_{k}\left(s_{j}^{\prime}, \beta_{j}^{\rho}\right)-L_{k}\left(s_{j}^{\prime}, \alpha_{j}^{\rho}\right)\right|<\varepsilon
$$

for every $j=1, \ldots, N$.

Remark 2.7. Using the subintervals of $\mathbb{R}_{+}^{N}$ instead of $\mathbb{R}^{N}$, consider the assumption (2.8). Then, following the proof of Proposition 3.5 in [5], one can show that, for a given kernel $L_{k}$, conditions (2.8) and (2.12) are equivalent.

Lemma 2.8. Assume that condition (2.12) (or, (2.8)) holds. If $f \in B V_{\mu}\left(\mathbb{R}_{+}^{N}\right)$, then $\mathcal{T}_{n, v}(f) \in A C_{\mu}\left(\mathbb{R}_{+}^{N}\right)$ for every $n, v \in \mathbb{N}$.

Proof. From Proposition 2.2 of [9] we immediately see that $\mathcal{T}_{n, v}(f) \in B V_{\mu}\left(\mathbb{R}_{+}^{N}\right)$. So, by the equivalence of absolute continuity and log-absolute continuity, it is enough to prove that, for a given $f \in B V_{\mu}\left(\mathbb{R}_{+}^{N}\right), \mathcal{T}_{n, v}(f)$ is log-absolutely continuous on every interval $I=\prod_{i=1}^{N}\left[a_{i}, b_{i}\right]$ of $\mathbb{R}_{+}^{N}$. Now for a given $\varepsilon>0$, fix a collection $\left\{\left[\alpha_{j}^{\rho}, \beta_{j}^{\rho}\right]\right\}_{\rho=1}^{\lambda}$ of nonoverlapping intervals in $\left[a_{j}, b_{j}\right]$ such that

$$
\sum_{\rho=1}^{\lambda}\left(\log \left(\beta_{j}^{\rho}\right)-\log \left(\alpha_{j}^{\rho}\right)\right)<\delta
$$

where the number $\delta$ comes from condition (2.12). Now letting $\mathbf{s t}=\mathbf{z}$, we get

$$
\mathcal{T}_{n, v}(f ; \mathbf{s})=\sum_{k=1}^{\infty} a_{n k}^{v} \int_{\mathbb{R}_{+}^{N}} L_{k}\left(\frac{\mathbf{z}}{\mathbf{s}}\right) H_{k}(f(\mathbf{z})) \frac{d \mathbf{z}}{\langle\mathbf{z}\rangle},
$$

where $\frac{\mathbf{z}}{\mathbf{s}}:=\left(\frac{z_{1}}{s_{1}}, \ldots, \frac{z_{N}}{s_{N}}\right)$. Since

$$
\sum_{\rho=1}^{\lambda}\left(\log \left(\beta_{j}^{\rho}\right)-\log \left(\alpha_{j}^{\rho}\right)\right)=\sum_{\rho=1}^{\lambda}\left|\log \left(z_{j} / \beta_{j}^{\rho}\right)-\log \left(z_{j} / \alpha_{j}^{\rho}\right)\right|<\delta
$$


it follows from the triangle inequality, condition (2.12) and the Fubini-Tonelli theorem that

$$
\begin{aligned}
\sum_{\rho=1}^{\lambda} \mid \mathcal{T}_{n, v}( & \left(f ; s_{j}^{\prime}, \beta_{j}^{\rho}\right)-\mathcal{T}_{n, v}\left(f ; s_{j}^{\prime}, \alpha_{j}^{\rho}\right) \mid \\
& \leq C \sum_{\rho=1}^{\lambda} \sum_{k=1}^{\infty} a_{n k}^{v} \int_{\mathbb{R}_{+}^{N}}\left|L_{k}\left(\frac{z_{j}^{\prime}}{s_{j}^{\prime}}, \frac{z_{j}}{\beta_{j}^{\rho}}\right)-L_{k}\left(\frac{z_{j}^{\prime}}{s_{j}^{\prime}}, \frac{z_{j}}{\alpha_{j}^{\rho}}\right)\right||f(\mathbf{z})| \frac{d \mathbf{z}}{\langle\mathbf{z}\rangle} \\
& =C \int_{\mathbb{R}_{+}^{N}} \sum_{\rho=1}^{\lambda} \sum_{k=1}^{\infty} a_{n k}^{v}\left|L_{k}\left(\frac{z_{j}^{\prime}}{s_{j}^{\prime}}, \frac{z_{j}}{\beta_{j}^{\rho}}\right)-L_{k}\left(\frac{z_{j}^{\prime}}{s_{j}^{\prime}}, \frac{z_{j}}{\alpha_{j}^{\rho}}\right)\right||f(\mathbf{z})| \frac{d \mathbf{z}}{\langle\mathbf{z}\rangle} \\
& \leq \varepsilon C\|f\|_{L_{\mu}^{1}},
\end{aligned}
$$

which completes the proof.

Theorem 2.9. Let $f \in B V_{\mu}\left(\mathbb{R}_{+}^{N}\right)$ and assume that conditions $(2.12),(i)^{\prime \prime}-(i i i)^{\prime \prime}$ and (iv) hold. Then

$$
f \in A C_{\mu}\left(\mathbb{R}_{+}^{N}\right) \Leftrightarrow \lim _{n \rightarrow \infty} V\left[\mathcal{T}_{n, v}(f)-f\right]=0 \text { uniformly in } v .
$$

Proof. If $f \in A C_{\mu}\left(\mathbb{R}_{+}^{N}\right)$, then by Theorem 2.3 of [9] we get

$$
\lim _{n \rightarrow \infty} V\left[\mathcal{T}_{n, v}(f)-f\right]=0 \text { uniformly in } v .
$$

Conversely, if we assume $\lim _{n \rightarrow \infty} V\left[\mathcal{T}_{n, v}(f)-f\right]=0$, since $A C_{\mu}\left(\mathbb{R}_{+}^{N}\right)$ is a closed subspace of $B V_{\mu}\left(\mathbb{R}^{N}\right)$ with respect to variation semi-norm (see [6]), we get from Lemma 2.8 that $f \in A C_{\mu}\left(\mathbb{R}_{+}^{N}\right)$.

\section{Characterizations of uniform continuity}

In this section, for the characterization of uniform continuity we use the supremum norm (uniform norm) instead of variation semi-norm. We consider the following three cases.

\subsection{Characterization in one dimension with periodicity}

We first recall some concepts.

- As usual, $C_{2 \pi}$ denotes the space of all $2 \pi$-periodic and continuous functions with the usual supremum norm $\|\cdot\|_{2 \pi}$

- $B_{2 \pi}$ denotes the space of all $2 \pi$-periodic and bounded functions.

For the nonlinear operators given by $(2.1)$, in addition to the conditions $(i)$ - (iii) stated in Subsection 2.1. it is needed to replace the condition (iv) with the following

(iv) ${ }^{\prime} \lim _{k \rightarrow \infty}\left\|G_{k}\right\|_{J}=0$ for every bounded interval $J \subset \mathbb{R}$, where $\|\cdot\|_{J}$ denotes the supremum norm on the interval $J$. Note that, this convergence does not need to be uniform with respect to $J \subset \mathbb{R}$.

We also assume the next condition.

For every $n, v \in \mathbb{N}$ and for all $\varepsilon>0$ there exists a $\delta>0$ such that

$$
|x-y|<\delta \text { implies } \sum_{k=1}^{\infty} a_{n k}^{v}\left|L_{k}(x)-L_{k}(y)\right|<\varepsilon .
$$

Lemma 3.1. Assume that (3.1) holds. If $f \in B_{2 \pi} \cap L_{2 \pi}^{1}$, then $\mathcal{T}_{n, v}(f) \in C_{2 \pi}$ for every $n, v \in \mathbb{N}$. 
Proof. As in the proof of Lemma 2.3, the operators (2.1) can be written as follows:

$$
\mathcal{T}_{n, v}(f ; z)=\sum_{k=1}^{\infty} a_{n k}^{v} \int_{-\pi}^{\pi} L_{k}(s-z) H_{k}(f(z)) d z .
$$

Using $H_{k}(0)=0$ it is not hard to see that

$$
\begin{aligned}
\left|\mathcal{T}_{n, v}(f ; y)-\mathcal{T}_{n, v}(f ; x)\right| & \leq C \sum_{k=1}^{\infty} a_{n k}^{v} \int_{-\pi}^{\pi}\left|L_{k}(y-z)-L_{k}(x-z)\right||f(z)| d z \\
& =C \int_{-\pi}^{\pi} \sum_{k=1}^{\infty} a_{n k}^{v}\left|L_{k}(y-z)-L_{k}(x-z)\right||f(z)| d z .
\end{aligned}
$$

Then, by (3.1), for all $x, y$ satisfying

$$
|x-y|=|x-z-(y-z)|<\delta
$$

we get

which gives the proof.

$$
\left|\mathcal{T}_{n, v}(f ; y)-\mathcal{T}_{n, v}(f ; x)\right| \leq C\|f\|_{1} \varepsilon
$$

The characterization theorem is the following.

Theorem 3.2. Let $f \in B_{2 \pi} \cap L_{2 \pi}^{1}$ and assume that conditions (3.1), (i) - (iii) and (iv $)^{\prime}$ hold. Then,

$$
f \in C_{2 \pi} \Leftrightarrow \lim _{n \rightarrow \infty}\left\|\mathcal{T}_{n, v}(f)-f\right\|_{2 \pi}=0 \text { uniformly in } v \text {. }
$$

Proof. The necessity is clear from Theorem 3.3 of [10]. The sufficiency part follows from Lemma 3.1 and the fact that $C_{2 \pi}$ is a closed subspace of $B_{2 \pi}$.

\subsection{Characterization of uniform continuity}

In this part, by $B U C\left(\mathbb{R}^{N}\right)$ we denote the space of all bounded and uniformly continuous functions on $\mathbb{R}^{N}$ endowed with the supremum norm $\|\cdot\|_{\infty}$. Then using the operators (2.5) we give a characterization of uniform continuity. Besides the conditions $(i)^{\prime}-(i i i)^{\prime}$ given in Subsection 2.2, we need $(i v)^{\prime}$ in Subsection 3.1. We also assume the next property.

For every $n, v \in \mathbb{N}$ and for all $\varepsilon>0$ there exists a $\delta>0$ such that

$$
|\mathbf{x}-\mathbf{y}|<\delta \text { implies } \sum_{k=1}^{\infty} a_{n k}^{v}\left|L_{k}(\mathbf{x})-L_{k}(\mathbf{y})\right|<\varepsilon \text {. }
$$

Lemma 3.3. Assume that (3.2) holds. If $f \in B\left(\mathbb{R}^{N}\right) \cap L^{1}\left(\mathbb{R}^{N}\right)$, then $\mathcal{T}_{n, v}(f) \in$ $B U C\left(\mathbb{R}^{N}\right)$ for every $n, v \in \mathbb{N}$.

Proof. From the proof of Lemma 2.5, we get

$$
\mathcal{T}_{n, v}(f ; \mathbf{z})=\sum_{k=1}^{\infty} a_{n k}^{v} \int_{\mathbb{R}^{N}} L_{k}(\mathbf{s}-\mathbf{z}) H_{k}(f(\mathbf{z})) d \mathbf{z} .
$$

Since $H_{k}(0)=0$, by the Fubini-Tonelli theorem, the inequality

$$
\begin{aligned}
\left|\mathcal{T}_{n, v}(f ; \mathbf{y})-\mathcal{T}_{n, v}(f ; \mathbf{x})\right| & \leq C \sum_{k=1}^{\infty} a_{n k}^{v} \int_{\mathbb{R}^{N}}\left|L_{k}(\mathbf{y}-\mathbf{z})-L_{k}(\mathbf{x}-\mathbf{z})\right||f(\mathbf{z})| d \mathbf{z} \\
& =C \int_{\mathbb{R}^{N}} \sum_{k=1}^{\infty} a_{n k}^{v}\left|L_{k}(\mathbf{y}-\mathbf{z})-L_{k}(\mathbf{x}-\mathbf{z})\right||f(\mathbf{z})| d \mathbf{z}
\end{aligned}
$$

holds. Considering the assumption, we observe that

$$
\left|\mathcal{T}_{n, v}(f ; \mathbf{y})-\mathcal{T}_{n, v}(f ; \mathbf{x})\right| \leq C\|f\|_{1} \varepsilon
$$

whenever $|\mathbf{x}-\mathbf{y}|=|\mathbf{x}-\mathbf{z}-(\mathbf{y}-\mathbf{z})|<\delta$. Here $\|f\|_{1}<\infty$ since $f \in L^{1}\left(\mathbb{R}^{N}\right)$. And finally by Lemma 3.1 of [11], we know that $\mathcal{T}_{n, v}(f) \in B\left(\mathbb{R}^{N}\right)$. Hence, the proof is completed. 
Theorem 3.4. Let $f \in B\left(\mathbb{R}^{N}\right) \cap L^{1}\left(\mathbb{R}^{N}\right)$ and assume that conditions $(3.2)$ and $(i)^{\prime}-(i v)^{\prime}$ hold. Then,

$$
f \in B U C\left(\mathbb{R}^{N}\right) \Leftrightarrow \lim _{n \rightarrow \infty}\left\|\mathcal{T}_{n, v}(f)-f\right\|_{\infty}=0 \text { uniformly in } v .
$$

Proof. For the necessity, apply Theorem 3.3 of [11]. For the sufficiency, use Lemma 3.3 and the fact that $B U C\left(\mathbb{R}^{N}\right)$ is a closed subspace of $B\left(\mathbb{R}^{N}\right)$.

\subsection{Characterization of log-uniform continuity}

Some notations and definitions used in this part are as follows.

- Let a function $f: \mathbb{R}_{+}^{N} \rightarrow \mathbb{R}$ be given. We say that $f$ is log-uniformly continuous on $\mathbb{R}_{+}^{N}$ if, for every $\varepsilon>0$ there is a $\delta>0$ such that, for all $\mathbf{x}, \mathbf{y} \in \mathbb{R}_{+}^{N}$ satisfying $|\log \mathbf{x}-\log \mathbf{y}|<\delta,|f(\mathbf{x})-f(\mathbf{y})|<\varepsilon$ holds, where $\log \mathbf{x}:=\left(\log x_{1}, \ldots, \log x_{N}\right)$. The space of all bounded and log-uniformly continuous functions on $\mathbb{R}_{+}^{N}$ is denoted by $B U C_{\log }\left(\mathbb{R}_{+}^{N}\right)$, which is endowed with the usual supremum norm $\|\cdot\|_{\infty}$.

- By $B\left(\mathbb{R}_{+}^{N}\right)$ we denote the space of all bounded functions on $\mathbb{R}_{+}^{N}$.

Notice that, in general, log-uniform continuity and uniform continuity are different concepts on $\mathbb{R}_{+}^{N}$; however they are equivalent on a bounded $N$-dimensional interval $\prod_{i=1}^{N}\left[a_{i}, b_{i}\right]$ of $\mathbb{R}_{+}^{N}$, where $b_{i} \geq a_{i}>0$ for all $i=1, \ldots, N$.

We use the assumptions $(i)^{\prime \prime}-(i i i)^{\prime \prime}$ in Subsection 2.3 and $(i v)^{\prime}$ in Subsection 3.1. We also need the next condition.

For every $n, v \in \mathbb{N}$ and for all $\varepsilon>0$, there exists $\delta>0$ such that

$$
|\log \mathbf{x}-\log \mathbf{y}|<\delta \text { implies } \sum_{k=1}^{\infty} a_{n k}^{v}\left|L_{k}(\mathbf{x})-L_{k}(\mathbf{y})\right|<\varepsilon .
$$

For the characterization theorem, we first prove the following two lemmas.

Lemma 3.5. Assume that (3.3) holds. If $f \in B\left(\mathbb{R}_{+}^{N}\right) \cap L_{\mu}^{1}\left(\mathbb{R}_{+}^{N}\right)$, then $\mathcal{T}_{n, v}(f) \in$ $B U C\left(\mathbb{R}_{+}^{N}\right)$ for every $n, v \in \mathbb{N}$.

Proof. After writing the operators (2.9) in the following form

$$
\mathcal{T}_{n, v}(f ; \mathbf{s})=\sum_{k=1}^{\infty} a_{n k}^{v} \int_{\mathbb{R}_{+}^{N}} L_{k}\left(\frac{\mathbf{z}}{\mathbf{s}}\right) H_{k}(f(\mathbf{z})) \frac{d \mathbf{z}}{\langle\mathbf{z}\rangle},
$$

we may write that

$$
\begin{aligned}
\left|\mathcal{T}_{n, v}(f ; \mathbf{y})-\mathcal{T}_{n, v}(f ; \mathbf{x})\right| & \leq C \sum_{k=1}^{\infty} a_{n k}^{v} \int_{\mathbb{R}_{+}^{N}}\left|L_{k}\left(\frac{\mathbf{z}}{\mathbf{y}}\right)-L_{k}\left(\frac{\mathbf{z}}{\mathbf{x}}\right)\right||f(\mathbf{z})| \frac{d \mathbf{z}}{\langle\mathbf{z}\rangle} \\
& =C \int_{\mathbb{R}_{+}^{N}} \sum_{k=1}^{\infty} a_{n k}^{v}\left|L_{k}\left(\frac{\mathbf{z}}{\mathbf{y}}\right)-L_{k}\left(\frac{\mathbf{z}}{\mathbf{x}}\right)\right||f(\mathbf{z})| \frac{d \mathbf{z}}{\langle\mathbf{z}\rangle}
\end{aligned}
$$

Then assumption (3.3) gives that, for a given $\varepsilon>0$, there is a $\delta>0$ such that, for all $\mathbf{x}, \mathbf{y}$ satisfying $|\log \mathbf{x}-\log \mathbf{y}|=\left|\log \frac{\mathbf{z}}{\mathbf{y}}-\log \frac{\mathbf{z}}{\mathbf{x}}\right|<\delta$, we get

$$
\left|\mathcal{T}_{n, v}(f ; \mathbf{y})-\mathcal{T}_{n, v}(f ; \mathbf{x})\right| \leq C\|f\|_{L_{\mu}^{1}} \varepsilon
$$

Combining this with Proposition 3.1 of [9], the proof is completed.

Lemma 3.6. $B U C_{\log }\left(\mathbb{R}_{+}^{N}\right)$ is a closed subspace of $B\left(\mathbb{R}_{+}^{N}\right)$ with respect to the supremum norm.

Proof. It is clear. 


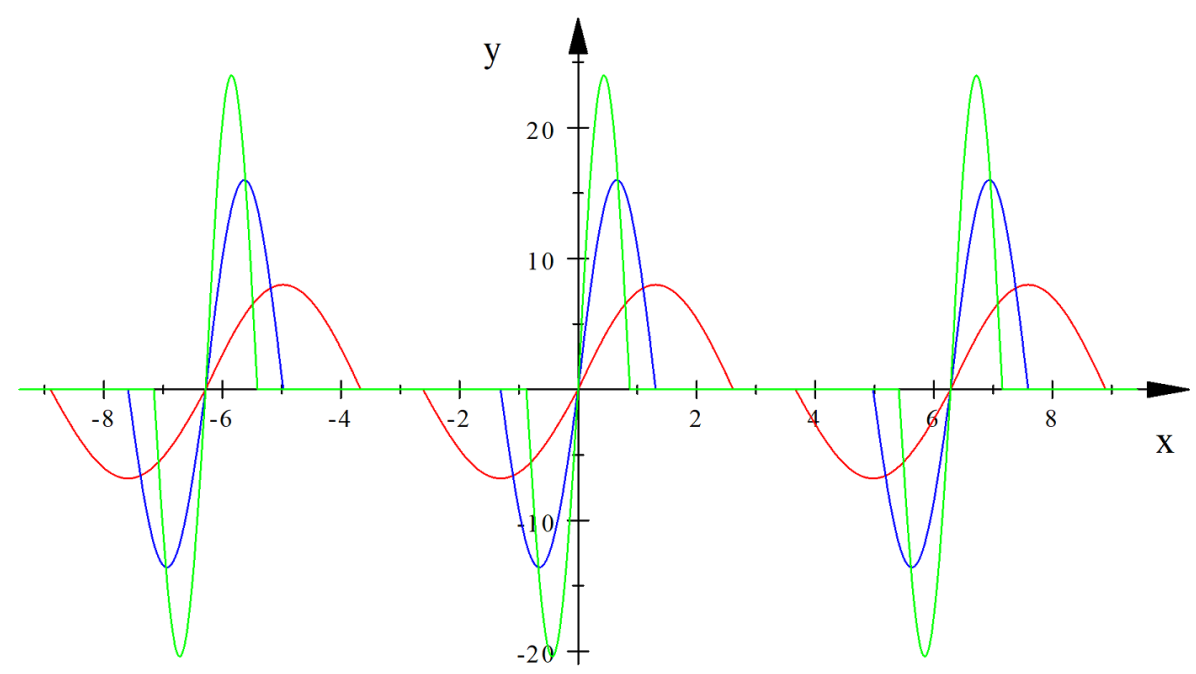

Figure 1. The kernel function $L_{k}$ given by (4.1) for $k=2,4,6$

Theorem 3.7. Let $f \in B\left(\mathbb{R}_{+}^{N}\right) \cap L_{\mu}^{1}\left(\mathbb{R}_{+}^{N}\right)$ and assume that $(3.3),(i)^{\prime \prime}-(i i i)^{\prime \prime}$ and $(i v)^{\prime}$ hold. Then,

$$
f \in B U C_{\log }\left(\mathbb{R}_{+}^{N}\right) \Leftrightarrow \lim _{n \rightarrow \infty}\left\|\mathcal{T}_{n, v}(f)-f\right\|_{\infty}=0 \text { uniformly in } v .
$$

Proof. Use Theorem 3.2 of [9] and Lemmas 3.5 and 3.6.

\section{Applications and special cases}

In this section, we give some suitable kernel functions satisfying our assumptions and also discuss some special cases of the characterization theorems.

Example 4.1. We first consider one dimensional case with periodicity. In the definition of the integral operators (2.1) and (2.2), take $\mathcal{A}=\left\{C_{1}\right\}=\left\{c_{n k}\right\}$ (Cesàro method) given by

$$
c_{n k}:= \begin{cases}\frac{1}{n} ; & k=1,2, \ldots, n \\ 0 ; & \text { otherwise }\end{cases}
$$

and also define the $2 \pi$-periodic kernel $L_{k}$ by

$$
L_{k}(t):= \begin{cases}\left((-1)^{k}+1\right) 2 k \sin \left(\frac{3 k t}{5}\right) ; & \text { if } 0 \leq t \leq \frac{5 \pi}{3 k} \\ \left((-1)^{k}+1\right) \frac{17}{10} k \sin \left(\frac{3 k t}{5}\right) ; & \text { if }-\frac{5 \pi}{3 k} \leq t<0 \\ 0 ; & \text { if } \pi \geq|t|>\frac{5 \pi}{3 k}\end{cases}
$$

which is extended by periodicity to the whole real line (see Figure 1).

In this case, observe that $L_{k}$ satisfies our conditions (i)-(iii) and (2.4). Furthermore, some suitable Lipschitz functions $H_{k}$ satisfying (iv) may be found in the papers [3, 4, 6, 911]. Hence, the corresponding operators are given by

$$
\mathcal{T}_{n}:=\mathcal{T}_{n, v}=\frac{1}{n} \sum_{k=1}^{n} T_{k}
$$

where

$$
T_{k}(f ; s)=\int_{-\pi}^{\pi} L_{k}(t) H_{k}(f(s-t)) d t
$$

Theorem 2.4 implies that

$$
f \in A C_{2 \pi} \Leftrightarrow \lim _{n \rightarrow \infty} V_{2 \pi}\left[\frac{T_{1}(f)+T_{2}(f)+\ldots+T_{n}(f)}{n}-f\right]=0 .
$$




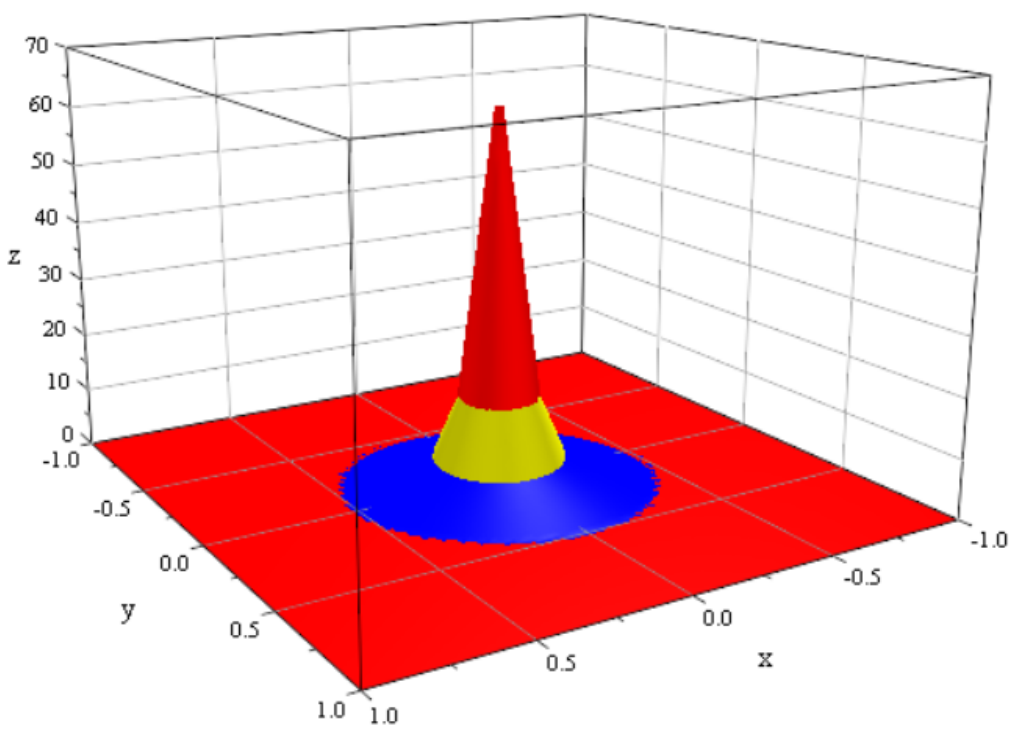

Figure 2. The kernel functions $L_{k}$ given by (4.4) for $k=2,4,6$

The limit on the right-hand side of (4.3) shows that the arithmetic mean of the sequence $\left\{T_{k}(f)\right\}$ in (4.2) converges to $f$ with respect to the variation semi-norm. However, one can observe that, for any nonconstant function $f$, the sequence $\left\{T_{k}(f)\right\}$ itself cannot be convergent to $f$ with respect to the variation semi-norm. Indeed, it follows from (4.1) and (4.2) that $L_{2 k-1}(t)=0$, which implies $T_{2 k-1}(f)=0$ for all $k \in \mathbb{N}$. Hence, we immediately get

$$
\lim _{k \rightarrow \infty} V_{2 \pi}\left[T_{2 k-1}(f)-f\right]=V_{2 \pi}[f] \neq 0
$$

for any nonconstant function $f$. So, the characterization in (4.3) is still valid although the sequence $\left\{T_{k}(f)\right\}$ in (4.2) does not converge to $f$ with respect to the variation semi-norm.

Example 4.2. Now take $\mathcal{A}=\mathcal{F}=\left\{c_{n k}^{v}\right\}$ (almost convergence method) given by

$$
c_{n k}^{v}:= \begin{cases}\frac{1}{n} ; & k=v, v+1, \ldots, v+n-1 \\ 0 ; & \text { otherwise }\end{cases}
$$

and define the kernel $L_{k}$ of two variables by

$$
L_{k}(x, y)= \begin{cases}\left((-1)^{k}+1\right) \frac{3 k^{3}}{\pi}\left(\frac{1}{k}-\sqrt{x^{2}+y^{2}}\right) ; & \text { if } \sqrt{x^{2}+y^{2}} \leq \frac{1}{k} \\ 0 ; & \text { if } \sqrt{x^{2}+y^{2}}>\frac{1}{k}\end{cases}
$$

which is indicated in Figure 2.

Then, it is easy to check that all conditions in Subsection 2.2 hold. Hence, for the corresponding operators

$$
\mathcal{T}_{n, v}=\frac{1}{n} \sum_{k=v}^{v+n-1} T_{k}
$$

where

$$
T_{k}(f ; s, t)=\int_{-\infty}^{\infty} \int_{-\infty}^{\infty} L_{k}(x, y) H_{k}(f(s-x, t-y)) d x d y,
$$

it follows from Theorem 2.6 that

$$
f \in A C\left(\mathbb{R}^{N}\right) \Leftrightarrow \lim _{n \rightarrow \infty} V\left[\frac{T_{v}(f)+T_{v+1}(f)+\ldots+T_{v+n-1}(f)}{n}-f\right]=0,
$$




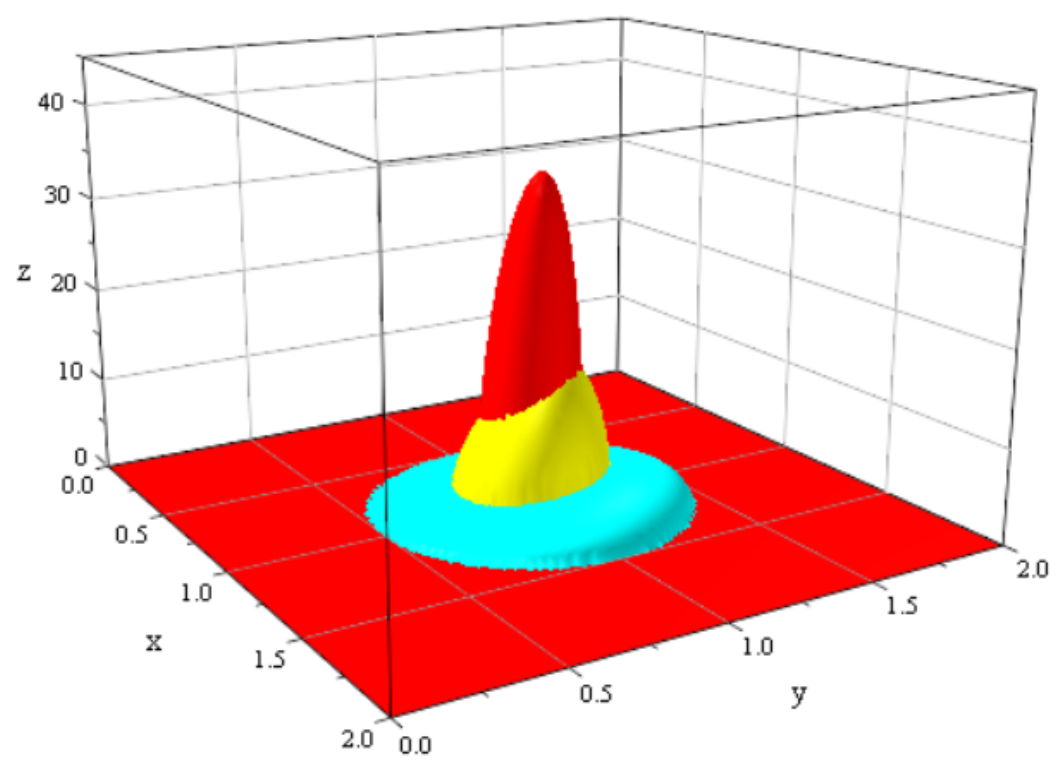

Figure 3. The kernel functions $L_{k}$ given by (4.7) for $k=2,4,6$

uniformly in $v$. As in Example 4.1, it is easy to see that

$$
\lim _{k \rightarrow \infty} V\left[T_{2 k-1}(f)-f\right]=V[f] \neq 0
$$

for any nonconstant function $f$. So, the characterization in (4.6) is still valid although the sequence $\left\{T_{k}(f)\right\}$ in (4.5) does not converge to $f$ with respect to the variation semi-norm.

Example 4.3. Finally, take again $\mathcal{A}=\mathcal{F}$ and define the kernel $L_{k}$ by

$$
L_{k}(x, y):= \begin{cases}\frac{3 k^{3} x y}{\pi} \sqrt{\frac{1}{k^{2}}-(x-1)^{2}-(y-1)^{2}} & ; \sqrt{(x-1)^{2}+(y-1)^{2}} \leq \frac{1}{k} \\ & \text { and } k=m^{2}, \\ \frac{3 k^{3} x y}{2 \pi} \sqrt{\frac{1}{k^{2}}-(x-1)^{2}-(y-1)^{2}} & ; \sqrt{(x-1)^{2}+(y-1)^{2}} \leq \frac{1}{k} \\ 0 & \text { and } k \neq m^{2} \\ & ; \text { otherwise, }\end{cases}
$$

which is indicated in Figure 3.

Then, for the corresponding operators

$$
\mathcal{T}_{n, v}=\frac{1}{n} \sum_{k=v}^{v+n-1} T_{k},
$$

where

$$
T_{k}(f ; s, t)=\int_{0}^{\infty} \int_{0}^{\infty} L_{k}(x, y) H_{k}(f(s-x, t-y)) \frac{d x d y}{x y},
$$

we may write from Theorem 2.9 that

$$
f \in A C_{\mu}\left(\mathbb{R}_{+}^{N}\right) \Leftrightarrow \lim _{n \rightarrow \infty} V\left[\frac{T_{v}(f)+T_{v+1}(f)+\ldots+T_{v+n-1}(f)}{n}-f\right]=0,
$$

uniformly in $v$.

One can also find suitable applications and some special cases (with respect to the supremum norm) of the results in Section 3. 


\section{References}

[1] G.A. Anastassiou and O. Duman, Towards Intelligent Modeling: Statistical Approximation Theory, Intelligent Systems Reference Library, vol. 14, Springer-Verlag, Berlin, 2011.

[2] L. Angeloni and G. Vinti, Convergence in variation and rate of approximation for nonlinear integral operators of convolution type, Results Math. 48, 1-23, 2006.

[3] L. Angeloni and G. Vinti, Erratum to: Convergence in variation and rate of approximation for nonlinear integral operators of convolution type, Results Math. 57, 387-391, 2010.

[4] L. Angeloni and G. Vinti, Variation and approximation in multidimensional setting for Mellin integral operators, New Perspectives on Approximation and Sampling Theory, 299-317, Appl. Numer. Harmon. Anal., Birkhäuser/Springer, Cham, 2014.

[5] L. Angeloni and G. Vinti, A characterization of absolute continuity by means of Mellin integral operators, Z. Anal. Anwend 34 (3), 343-356, 2015.

[6] L. Angeloni and G. Vinti, Convergence in variation and a characterization of the absolute continuity, Integral Transforms Spec. Funct. 26, 829-844, 2015.

[7] L. Angeloni and G. Vinti, A concept of absolute continuity and its characterization in terms of convergence in variation, Math. Nachr. 289 (16), 1986-1994, 2016.

[8] I. Aslan and O. Duman, A summability process on Baskakov-type approximation, Period. Math. Hungar. 72 (2), 186-199, 2016.

[9] I. Aslan and O. Duman, Summability on Mellin-type nonlinear integral operators, Integral Transforms Spec. Funct. 30 (6), 492-511, 2019.

[10] I. Aslan and O. Duman, Approximation by nonlinear integral operators via summability process, Math. Nachr., 2020 (in press) doi: 10.1002/mana.201800187.

[11] I. Aslan and O. Duman, Nonlinear approximation in $N$-dimension with the help of summability methods, submitted for publication.

[12] Ö.G. Atlihan and C. Orhan, Summation process of positive linear operators, Comput. Math. Appl. 56, 1188-1195, 2008.

[13] C. Bardaro, P.L. Butzer and I. Mantellini, The exponential sampling theorem of signal analysis and the reproducing kernel formula in the Mellin transform setting, Sampl Theory Signal Image Process. 13 (1), 35-66, 2014.

[14] C. Bardaro, P.L. Butzer, R.L. Stens and G. Vinti, Convergence in variation and rates of approximation for Bernstein-type polynomials and singular convolution integrals, Analysis 23, 299-340, 2003.

[15] H.T. Bell, $\mathcal{A}$-summability, Dissertation, Lehigh University, Bethlehem., Pa., 1971.

[16] H.T. Bell, Order summability and almost convergence, Proc. Amer. Math. Soc. 38, 548-552, 1973.

[17] M. Bertero and E.R. Pike, Exponential-sampling method for Laplace and other dilationally invariant transforms. II. Examples in photon correlation spectroscopy and Fraunhofer diffraction, Inverse Probl. 7 (1), 21-41, 1991.

[18] J. Boos, Classical and Modern Methods in Summability, Oxford University Press, Oxford, 2000.

[19] P.L. Butzer and S. Jansche, A direct approach to the Mellin transform, J. Fourier Anal. Appl. 3 (4), 325-376, 1997.

[20] P.L. Butzer and S. Jansche, A self-contained approach to Mellin transform analysis for square integrable functions; applications, Integral Transforms Spec. Funct. 8 (3-4), 175-198, 1999.

[21] P.L. Butzer and R.J. Nessel, Fourier Analysis and Approximation, Lehrbücher und Monographien aus dem Gebiete der exakten Wissenschaften, Mathematische Reihe 40, Birkhäuser, Basel, Academic Press, New York, 1971. 
[22] D. Casasent, Optical signal processing, in: Optical Data Processing, 241-282, Springer, Berlin, 1978.

[23] A. De Sena and D. Rocchesso, A fast Mellin and scale transform, EURASIP J. Adv. Signal Process, Art. ID 89170, 9 pages, 2007.

[24] G.H. Hardy, Divergent Series, Oxford University Press, Oxford, 1949.

[25] W.B. Jurkat and A. Peyerimhoff, Fourier effectiveness and order summability, J. Approx. Theory 4, 231-244, 1971.

[26] W.B. Jurkat and A. Peyerimhoff, Inclusion theorems and order summability, J. Approx. Theory 4, 245-262, 1971.

[27] G.G. Lorentz, A contribution to the theory of divergent sequences, Acta Math. 80, 167-190, 1948.

[28] R.G. Mamedov, The Mellin Transform and Approximation Theory, Elm, Baku, 1991.

[29] R.N. Mohapatra, Quantitative results on almost convergence of a sequence of positive linear operators, J. Approx. Theory 20, 239-250, 1977.

[30] D.A. Smith and W.F. Ford, Acceleration of linear and logarithmical convergence, Siam J. Numer. Anal. 16, 223-240, 1979.

[31] J.J. Swetits, On summability and positive linear operators, J. Approx. Theory 25, 186-188, 1979.

[32] L. Tonelli, Su alcuni concetti dell'analisi moderna, Ann. Scuola Norm. Super. Pisa 11 (2), 107-118, 1942.

[33] J. Wimp, Sequence Transformations and Their Applications, Academic Press, New York, 1981. 